\title{
Dialysis Dose and Risk Factors for Death Among ESRD Patients Treated with Twice-Weekly Hemodialysis: A Prospective Cohort Study
}

\author{
Thanachai Panaput $^{a}$ Bandit Thinkhamrop ${ }^{a}$ Somnuek Domrongkitchaiporn ${ }^{b}$ \\ Dhavee Sirivongs $^{c}$ Laksamon Praderm ${ }^{\mathrm{e}}$ Jirasak Anukulanantachai ${ }^{\mathrm{d}}$ \\ Chavasak Kanokkantapong $^{f}$ Pakorn Tungkasereerak ${ }^{g}$ Cholatip Pongskulc \\ Sirirat Anutrakulchaic Thathsalang Keobounma ${ }^{\text {h }}$ Surapong Narenpitak ${ }^{j}$ \\ Pisith Intarawongchot ${ }^{\mathrm{i}}$ Ammrit Suwattanasink Sajja Tatiyanupanwong' \\ Kannika Niwattayakul ${ }^{m}$ \\ ${ }^{a}$ Department of Biostatistics and Demography, Faculty of Public Health, Khon Kaen University, Khon Kaen, \\ ${ }^{\mathrm{b}}$ Department of Medicine, Ramathibodi Hospital, Mahidol University, Bangkok, 'Department of Medicine, Srinagarind \\ Hospital, Khon Kaen University, ${ }^{d}$ Khon Kaen Hospital, Khon Kaen, ${ }^{e}$ Roi Et Hospital, Roi Et, ${ }^{\text {} M a h a r a t ~ N a k h o n ~ R a t c h a s i m a ~}$ \\ Hospital, Nakhon Ratchasima, ${ }^{9}$ Surin Hospital, Surin, ${ }^{\text {h}}$ Thabo Crown Prince Hospital and 'Nong Khai Hospital, \\ Nong Khai, 'Udon Thani Hospital, Udon Thani, 'kMahasarakham Hospital, Mahasarakham, 'Chaiyaphum Hospital, \\ Chaiyaphum, and ${ }^{\mathrm{m}}$ Loei Hospital, Loei, The HENNET Study Group, Thailand
}

\section{Key Words}

Adequacy · Hemodialysis · spKt/V · Prognostic factors ·

Twice-weekly dialysis

\begin{abstract}
Background/Aims: We aimed to define the dosing and risk factors for death in patients undergoing twice-weekly hemodialysis. Methods: A prospective multi-center cohort study was conducted with one-year observation. Patients treated with twice- or thrice-weekly hemodialysis were identified. Death and first admission were the outcomes. spKt/V was a factor of interest. Results: We enrolled 504 twiceweekly and 169 thrice-weekly hemodialysis patients. The mean weekly values of spKt/V in the two groups were 3.4 and 5.1. The one-year survival rate and times to hospitalization were similar in both groups. The hazard ratios for death in higher spKt/V quartile was not associated with lower mortal-
\end{abstract}

\section{KARGER}

E-Mail karger@karger.com

www.karger.com/bpu ity, $p=0.70$. The four significant predictors for death were serum albumin, $H R=2.6$, current smoking, $H R=19.3$, age, $H R=1.1$, and the Index of Coexistent Disease [ICED], $H R=1.9$. Conclusion: The effect of spKt/V on short-term mortality was not obvious in twice-weekly dialysis patients. Attention should be paid to patients who smoke, have hypoalbuminemia, are elderly, or have a high ICED. ๑ 2015 S. Karger AG, Basel

\section{Introduction}

Thrice-weekly hemodialysis (HD) has been considered the standard dialysis frequency for most end-stage renal disease (ESRD) patients requiring $\mathrm{HD}$ [1]. It is recommended that twice-weekly HD should be prescribed only to patients with residual renal urea clearance of more than $2 \mathrm{ml} / \mathrm{min} / 1.73 \mathrm{~m}^{2}$ and the minimum dialysis dose 
should be a spKt/V of 2 or more per dialysis. The recommended dialysis dosage for twice-weekly HD is based on a value derived from thrice-weekly HD kinetics [1]. Studies to determine the effect of spKt/V on mortality and other predictors of mortality among HD patients have been conducted almost exclusively in thrice-weekly HD [2-4]. However, twice-weekly HD is still prevalent in resource-limited setting, mostly in developing countries, and occasionally in Europe and in the United States $[5,6]$.

In Thailand, patients with ESRD under the coverage of the Social Health Security Program are only financially supported for only twice-weekly HD. In this unfortunate situation, the majority of Thai patients with ESRD receive twice-weekly HD. The situation provides an opportunity to study the effect on mortality as well as other predictors of death in twice-weekly HD. This study, therefore, aimed to evaluate the short-term outcome of twice-weekly HD and determine the effects of spKt/V and other risk factors for death among patients treated with twice-weekly HD.

\section{Methods}

\section{Study Design}

A prospective multi-centered cohort design was used. Participants were selected from 11 dialysis centers in northeastern Thailand. The accrual period was between February and May 2011, and the participants were followed until April 30, 2012.

\section{Participants}

ESRD patients aged between 18 and 80 who had been treated with twice-weekly HD for more than three months were enrolled in this study. Exclusion criteria were patients who were pregnant, those with a psychiatric problem, those who were bed-ridden, and those receiving either chemotherapy or radiation for malignancy. Participants who had previously received peritoneal dialysis prior to HD therapy were also excluded. Patients' dry weight, dose of heparin, blood pressure and metabolic control, and additional intra-dialysis treatments, such as intravenous iron therapy or dosage of epoietin, were adjusted by the attending nephrologist. Patients who had been on thrice-weekly HD were also recruited for comparison of outcomes. The latter group of patients was managed under the same therapeutic regime and followed up as was done in the twice-weekly HD group; the difference was only in the number of dialysis sessions.

\section{Data Collections}

Demographic and socioeconomic data, starting date of dialysis, primary renal disease, dialysis prescribing data including dialysis time, dialyzer membrane, flux and number of dialyzer reuse were obtained using standardized records by study personnel at entry to the study. The Index of Coexistent Disease [ICED] [7, 8] was used to assess comorbidity at recruitment. It comprised of two separate sections including the Index of Disease Severity [IDS] and the Index of Physical Impairment [IPI]. We used the modified version suggested by the HEMO study group [9]. The health person- nel who participated in this study from each center were trained to gather IDS and IPI. The IDS and IPI category scores were used to determine the ICED levels ( 0 to 3 ), which were scored by one dedicated research nurse [9]. The estimated glomerular filtration rate (eGFR) before the start of dialysis therapy was calculated using the Chronic Kidney Disease Epidemiology Collaboration (CKD-EPI) formula $[10,11]$.

Pre-dialysis and post-dialysis blood pressure and biochemical studies were recorded at the start of study and then twice more at two monthly intervals. Dialysis dose, determined by using spKt/V, was calculated from pre- and post-dialysis urea concentrations based on K-DOQI guideline [1]. SpKt/V and laboratory studies included hemoglobin, serum creatinine, electrolytes, calcium, phosphorus, serum albumin, and the like were also recorded at the start of study and then twice more at two monthly intervals. The average values of the three measurements of these parameters were used as input for analysis. The most recent values of parameters before recruitment, including low-density lipoprotein cholesterol [LDL], triglyceride, intact parathyroid hormone, and ferritin level, were recorded. Viral markers including hepatitis B surface antigen, hepatitis $\mathrm{C}$ antibody, and human immunodeficiency virus antibody were determined. Self-reported urine output of each participant was recorded. Anuria was defined as urine output less than $100 \mathrm{ml}$ per day.

\section{Outcomes}

The main outcome was the time to disease-related death. The identification of the associated causes of death was performed by attending nephrologists. Alternative relevant clinical outcomes regarding kidney transplantation, switch to peritoneal dialysis, change frequency of HD session, withdrawal from therapy, or transfer to other centers were recorded. The secondary outcome was the time to first hospitalization of any cause.

\section{Statistical Analysis}

The sample size was calculated for one group survival analysis following the formula suggested by Schmoor et al. [12]. A two-side test with an overall sample size of 456 participants, of whom 41 died, achieved $80 \%$ power at a 0.05 significant level to detect a hazard ratio [HR] of 2.5 [13]. This assumed that the proportion of the factor of interest, $\mathrm{spKt} / \mathrm{V}<2$, was 0.44 and the correlation between this factor and other covariates (rho) is 0.27 , giving a variation inflation factor of 1.08 . After allowing for a $10 \%$ of drop out, the total number recruited was 507.

Patient survival was related to multiple risk factors. Continuous variables were categorized according to the cut-off points commonly used in previous studies and those in which their medians were used as cut-off points, were shown in the appendix. Continuous variables including age, income, body mass index, time on dialysis, number of dialyzer reuse, serum intact parathyroid hormone level, and spKt/V were divided into quartile ranges. Other potential risk factors included currently smoking, anuria, semi-synthetic dialyzer, low flux dialyzer, mean arterial pressure pre-dialysis of $>100 \mathrm{~mm} \mathrm{Hg}$, mean arterial pressure post-dialysis of $>100 \mathrm{~mm} \mathrm{Hg}$, cardiac arrhythmia or abnormal electrocardiography (ECG), aspirin use, statin use, angiotensin converting enzyme inhibitor [ACEI] or angiotensin receptor blocker [ARB] use, the presence of hepatitis $B$ surface antigen, and the presence of hepatitis $\mathrm{C}$ antibody. The dates of the endpoint, death were recorded. 
Continuous data were presented as mean \pm standard deviation [SD] or median (inter-quartile range). Categorical data were presented as proportions. The Kaplan-Meier survival method was used to estimate survival probability. The start of the study was the date of enrollment and the end of study was the date when patients were dead or alive and were still treated with continuous HD. Univariate analysis of the risk factors was done using univariate Cox regression analysis. Multivariable Cox regression analysis was performed to determine the effect of spKt/V on mortality and to identify the predictors of mortality, taking into account the effects of potential factors with $p$-value of $<0.2$ obtained from univariate analysis. Linear relationships between continuous variables and mortality were explored. The significance of each variable in the model was computed by using the likelihood ratio test. Model assumptions and goodness of fit to data were assessed for the final model. The hazard ratios and their 95\% confidence intervals [CI] adjusted for time on dialysis and dialysis centers were estimated.

This study was approved by the institutional review board of the Ministry of Public Health and also by the institutional review board of the Khon Kaen University. Written informed consent was obtained from all participants.

\section{Results}

\section{Study Participants}

Five hundred and four patients were being treated with twice-weekly HD. Sixty-one (12.1\%) participants were incident patients, having been on $\mathrm{HD}$ for $<1$ year, and the other 443 were prevalent patients, having been on $\mathrm{HD}$ for $>1$ year at time of enrollment into the study. There were 169 patients treated with thrice-weekly HD; table 1.

\section{Outcomes and Survival Analysis}

Among the twice-weekly dialysis group, 6,045 patientmonths were observed (median time, 12.8 months). During the study period, $32(6.3 \%, 95 \% \mathrm{CI} ; 4.2$ to 8.5$)$ patients died. The characteristics of the patients classified by outcomes are presented in table 1 . The overall mortality rate was 5.8 deaths $/ 100$ person-years (95\% CI; 4.1 to 8.0 ). The overall probability of survival after one year was 0.94 ( $95 \%$ CI; 0.92 to 0.96 ). There was no significant difference in the mortality rate between genders. The characteristics of the patients and associated causes of death in the incident and prevalent groups are shown in table 2. Other non-fatal clinical events were: (1) transferring to another dialysis centers in twenty-seven (5.4\%) patients; (2) increase in frequency of dialysis to thrice-weekly, in thirteen $(2.6 \%)$ patients; (3) switching to peritoneal dialysis mode in ten (2\%) patients; (4) kidney transplantation in six (1.2\%) patients, and (5) withdrawal from therapy in six $(1.2 \%)$ patients. The one-year survival among the in- cident patients, whom were on dialysis therapy for 3 to 12 months, 0.98 ( $95 \%$ CI; 0.89 to 0.99 ), was not significantly different from that of the prevalent patients, 0.94 (95\% CI; 0.91 to 0.96 ), log-rank test, $\mathrm{p}=0.33$.

Among the thrice-weekly group, 2,081 patientmonths were observed. Eleven (6.5\%, 95\% CI; 2.8 to 10.3$)$ patients died. The one-year survival of patients in the thrice-weekly HD group, 0.94 (95\% CI; 0.89 to 0.96 ), was comparable to that of the twice-weekly group, log-rank test, $\mathrm{p}=0.93$ (fig. 1 ). The major causes of death among the thrice-weekly group were cardiovascular complications and infections (table 3 ). There were more patients with diabetes mellitus and who had a longer time on dialysis in the thrice-weekly HD group compared to the twice-weekly HD group (table 1). However, the hazard ratio for death adjusted for diabetes mellitus and the duration of HD before entry of the twice-weekly HD group was close to 1 when compared to those of the thriceweekly $\mathrm{HD}$ group $(\mathrm{HR}=0.99,95 \% \mathrm{CI} ; 0.47$ to $2.08, \mathrm{p}=$ 0.98).

\section{First Hospitalization}

Of total 673 patients, 7,002 patient-months of first admission were observed. There were 5,273 patient-months observed in the twice-weekly group and 1,929 patientmonths in the thrice-weekly group. There were 128 (25.4\%) patients in the twice-weekly HD group and 37 (21.9\%) in the thrice-weekly HD group who needed first hospitalization within one year. The probability of patients not requiring hospitalization within one year for the twice-weekly HD group, 0.76 (95\% CI; 0.72 to 0.80$)$, was comparable to that of the thrice-weekly HD group, 0.79 (95\% CI; 0.72 to 0.84 ), log-rank test $\mathrm{p}=0.22$ (fig. 2). The causes of hospitalization in the two groups were comparable, Fisher's exact $=0.37$. The two most common causes, accounting for more than $50 \%$, were infection related and cardiovascular disorders (table 4).

\section{spKt/V and Mortality}

In the twice-weekly HD group, the spKt/V was $1.7 \pm$ 0.3 (median 1.7, range 0.67 to 2.83 ). There was a significant difference in the spKt/V between genders, $1.9 \pm 0.3$ for females and $1.6 \pm 0.3$ for males, with a mean difference of $0.3,95 \% \mathrm{CI} ; 0.23$ to $0.34, \mathrm{p}<0.001$. The characteristics and death rates according to spKt/V quartiles are shown in table 4 . The unadjusted hazard ratio (HR) for death and the HR adjusted for gender by quartiles tended to be lower for patients in the higher quartiles; however, the differences which were $p=0.70$ and 0.18 , respectively were not significant. 
Table 1. Characteristics of patients with end-stage renal disease classified by frequency of dialysis

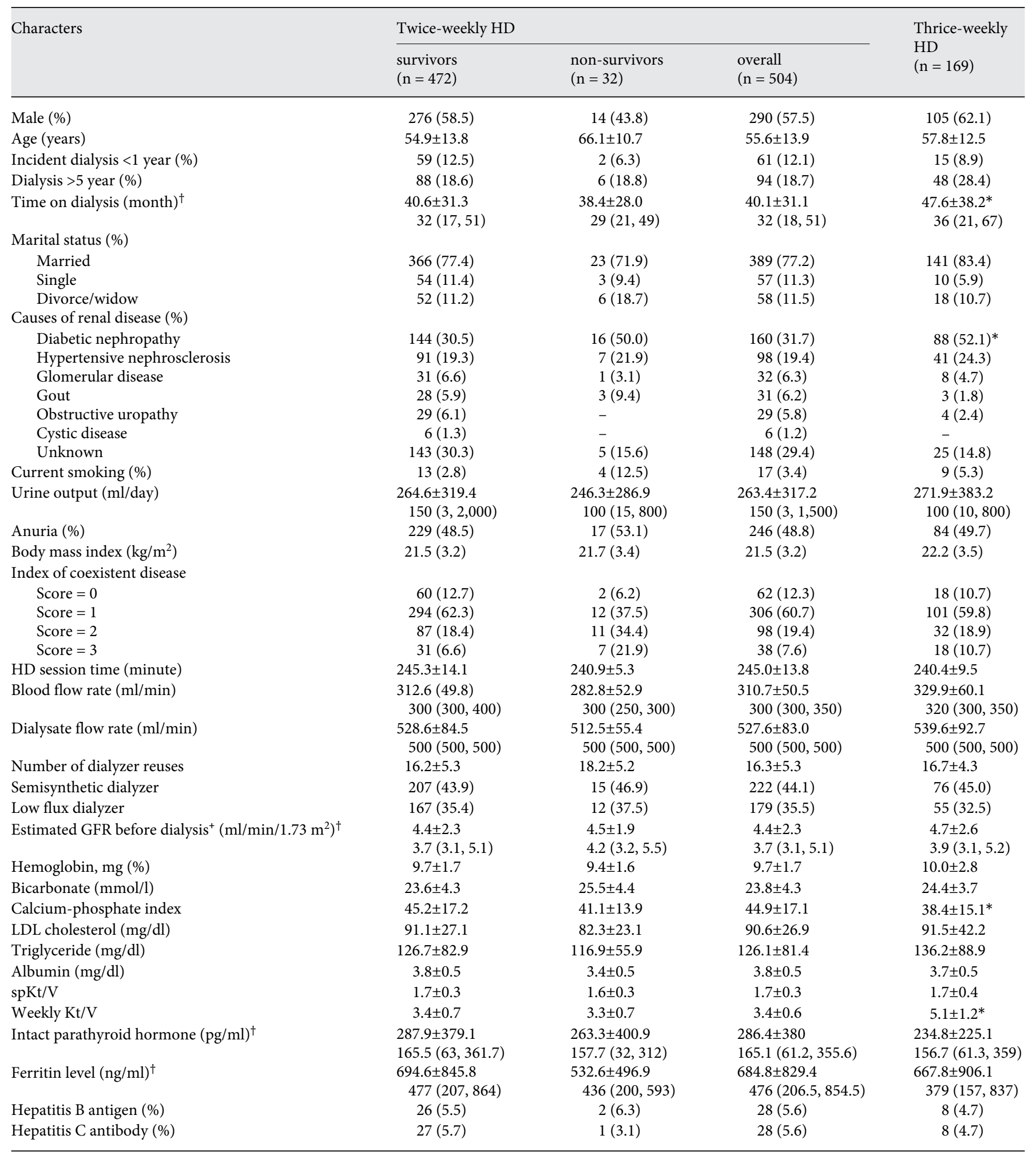

${ }^{*} \mathrm{p}<0.05$ when compared to overall twice-weekly group; ${ }^{\dagger}$ mean \pm standard deviation, median (inter-quartile range); ${ }^{+}$using Chronic Kidney Disease Epidemiology Collaboration (CKD-EPI) formula. 
Table 2. Characteristics and causes of death in incident patients, prevalent patients, twice-weekly dialysis-, and thrice-weekly dialysis patients

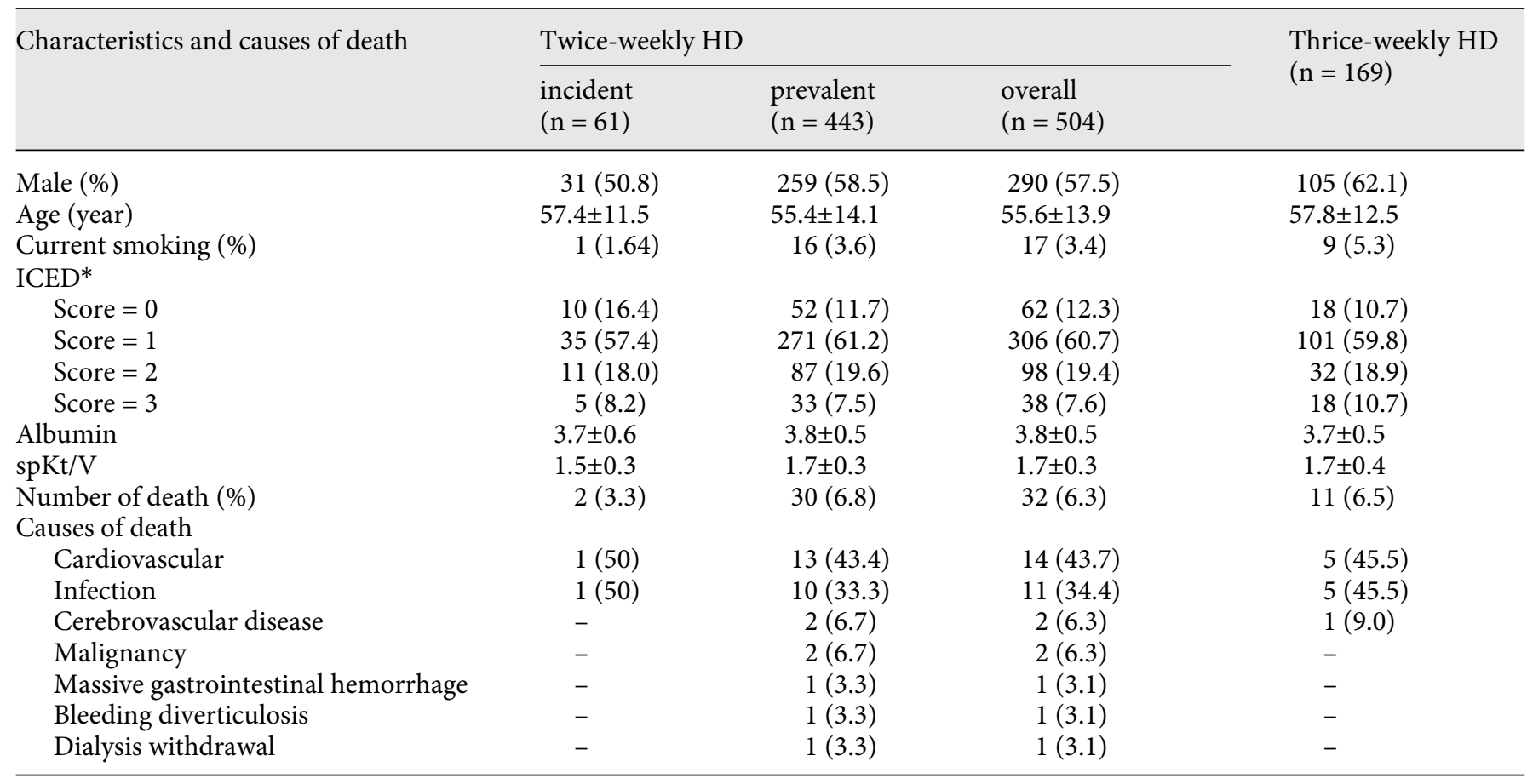

* Index of coexistent disease.

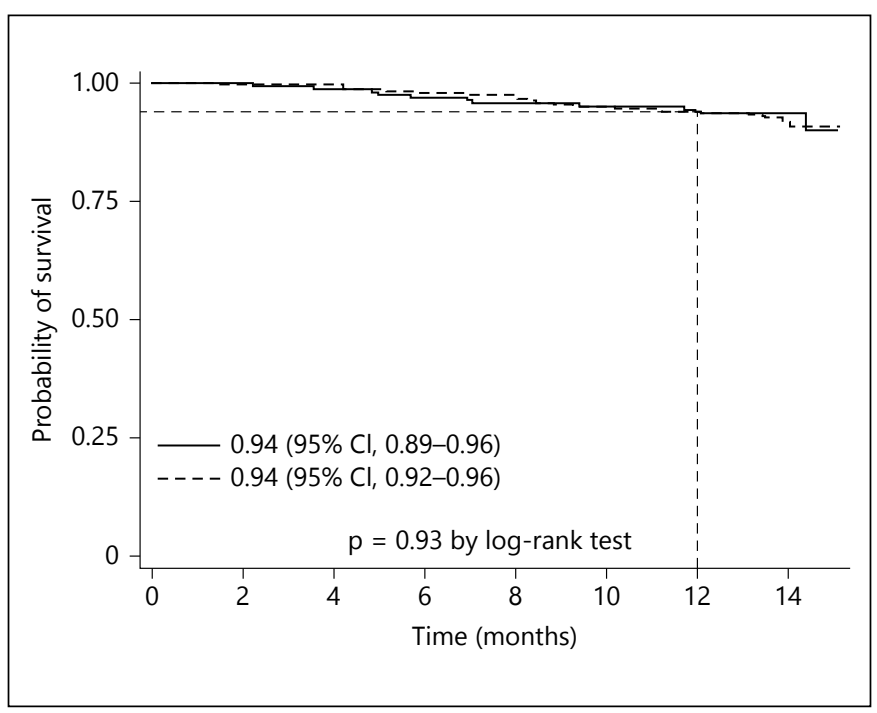

Fig. 1. Kaplan-Meier estimates of survival in patients treated with twice-weekly and thrice-weekly hemodialysis. Shown are estimates of survival among patients treated with thrice-weekly hemodialysis (solid line) and those with twice-weekly hemodialysis (broken line). Numbers are one-year survival probabilities and 95\% confidence intervals.

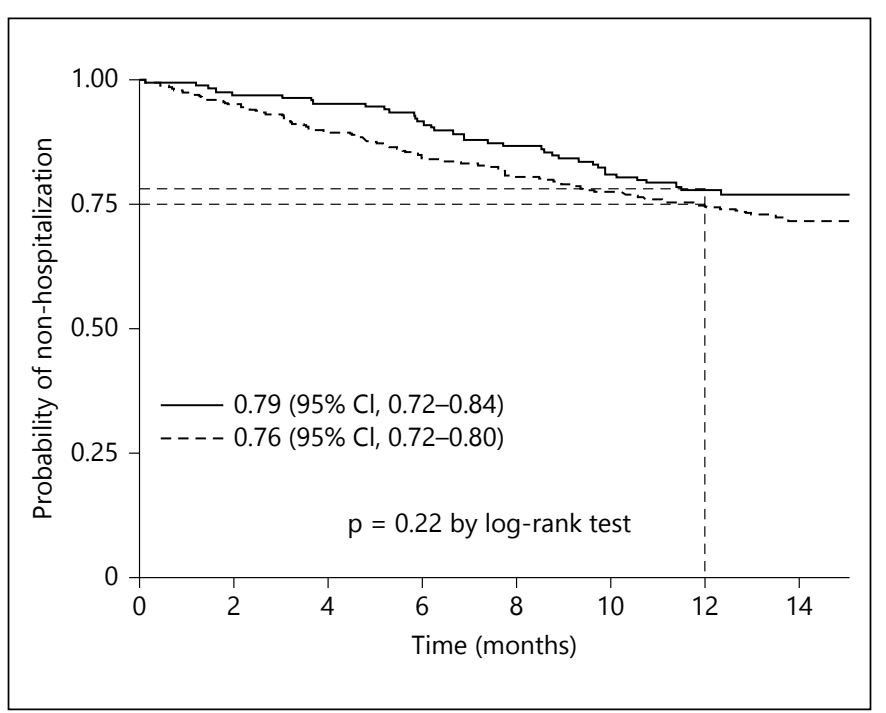

Fig. 2. Kaplan-Meier estimates of patients who did not need hospitalization in the twice-weekly and the thrice-weekly hemodialysis groups. Shown are estimates of non-hospitalization among patients treated with thrice-weekly hemodialysis (solid line) and those with twice-weekly hemodialysis (broken line). Numbers are one year probabilities of non-hospitalization and $95 \%$ confidence intervals. 
Table 3. Death rates and hazard ratios by spKt/V quartiles in patients treated with twice-weekly dialysis

\begin{tabular}{|c|c|c|c|c|c|}
\hline \multirow[t]{2}{*}{ Characteristics } & \multicolumn{4}{|c|}{ spKt/V quartiles } & \multirow[t]{2}{*}{$\mathrm{p}$} \\
\hline & $\begin{array}{l}0.67-1.45 \\
(\mathrm{n}=134)\end{array}$ & $\begin{array}{l}1.46-1.67 \\
(\mathrm{n}=124)\end{array}$ & $\begin{array}{l}1.68-1.90 \\
(\mathrm{n}=121)\end{array}$ & $\begin{array}{l}1.91-2.83 \\
(\mathrm{n}=125)\end{array}$ & \\
\hline Male (\%) & $107(79.9)$ & $86(69.4)$ & $64(52.9)$ & $33(26.4)$ & $<0.01$ \\
\hline Age (years) & $55.7 \pm 13.4$ & $56.1 \pm 13.9$ & $56.8 \pm 13.3$ & $53.9 \pm 14.8$ & 0.39 \\
\hline Current smoking (\%) & $5(3.7)$ & $7(41.2)$ & $4(23.5)$ & $1(5.9)$ & 0.17 \\
\hline ICED* & & & & & 0.30 \\
\hline Score $=0$ & $14(10.5)$ & $13(10.5)$ & $18(14.9)$ & $17(13.6)$ & \\
\hline Score $=1$ & $85(63.4)$ & $78(62.9)$ & $63(52.1)$ & $80(64.0)$ & \\
\hline Score $=2$ & $29(21.6)$ & $24(19.3)$ & $29(23.9)$ & $16(12.8)$ & \\
\hline Score $=3$ & $6(4.5)$ & $9(7.3)$ & $11(9.1)$ & $12(9.6)$ & \\
\hline Albumin (g/dl) & $3.7 \pm 0.6$ & $3.8 \pm 0.5$ & $3.8 \pm 0.5$ & $3.8 \pm 0.5$ & 0.73 \\
\hline Death $(\%)$ & $10(7.5)$ & $9(7.3)$ & $7(5.8)$ & $6(4.8)$ & 0.79 \\
\hline $\mathrm{HR}(95 \% \mathrm{CI})^{\dagger}$ & Reference & $1(0.4-2.6)$ & $0.8(0.3-2.0)$ & $0.6(0.2-1.7)$ & 0.70 \\
\hline HRadj $(95 \% \mathrm{CI})^{+}$ & Reference & $0.9(0.4-2.3)$ & $0.6(0.2-1.6)$ & $0.4(0.1-1.2)$ & 0.18 \\
\hline
\end{tabular}

* Index of coexistent disease; ${ }^{\dagger}$ Unadjusted hazard ratio (95\% confidence interval); ${ }^{+}$Hazard ratio adjusted for gender ( $95 \%$ confidence interval).

Table 4. Causes of hospitalization during the period of observation in twice-weekly and thrice-weekly dialysis patients

\begin{tabular}{lcc}
\hline Causes of hospitalization & $\begin{array}{l}\text { Twice-weekly HD } \\
(\mathrm{n}=128)\end{array}$ & $\begin{array}{l}\text { Thrice-weekly HD } \\
(\mathrm{n}=37)\end{array}$ \\
\hline Infection & $39(30.5)$ & $13(35.2)$ \\
Cardiovascular disorder & $34(26.5)$ & $7(18.9)$ \\
Vascular access problem & $10(7.8)$ & $3(8.1)$ \\
Gastrointestinal or other hemorrhage & $7(5.5)$ & $2(5.4)$ \\
Cerebrovascular disease & $4(3.1)$ & $5(13.5)$ \\
Severe hypertension & $6(4.7)$ & $1(2.7)$ \\
Anemic symptom & $6(4.7)$ & - \\
Potassium disorder & $4(3.1)$ & $1(8.1)$ \\
Others & $3(2.3)$ & - \\
Musculoskeletal disorder & $3(2.3)$ & - \\
Malignancy & $3(2.3)$ & $1(2.7)$ \\
Severe dyspepsia & $2(1.6)$ & - \\
Renal calculi/obstructive uropathy & $2(1.6)$ & $1(2.7)$ \\
Parathyroidectomy & $2(1.6)$ & - \\
Severe vertigo & - & - \\
Depression & $1(0.8)$ & - \\
Snake bite & $1(0.8)$ & $1(0.8)$ \\
Hypoglycemia & & \\
Arterial occlusion & & \\
\hline
\end{tabular}

Prognostic Factors among Twice-Weekly HD Group

Univariate Cox regression analysis was performed and twelve risk factors associated with death $(\mathrm{p}<0.2)$ were identified, including age, male gender, current smoking, ICED, number of dialyzer reuses, aspirin usage, statin usage, ACEI or ARB usage, creatinine $<11.7 \mathrm{mg} / \mathrm{dl}$, serum albumin $<3.5 \mathrm{~g} / \mathrm{dl}$, potassium $>5 \mathrm{mmol} / \mathrm{l}$ and bicarbonate $>24 \mathrm{mmol} / \mathrm{l}$. After applying the multivariate Cox regression analysis, adjusted for time on HD and HD centers, four independent predictors were identified, including current smoking, HR 19.3 (95\% CI; 4.8 to 76.9 , p < 0.001), serum albumin level, HR 2.6 for every $1 \mathrm{~g} / \mathrm{dl}$ decrease 
Fig. 3. Hazard ratio for death derived from $\mathrm{spKt} / \mathrm{V}$ and independent predictors. Hazard ratios for death from spKt/V and 95\% confidence intervals (CI) in patients who were treated with twice-weekly hemodialysis which were adjusted for age, time on hemodialysis, ICED (Index of Coexistent Disease), albumin level, current smoking and dialysis center. This model was tested for goodness of fit, $p=0.39$ and was not against the proportional hazard assumption, $\mathrm{p}=0.74$.

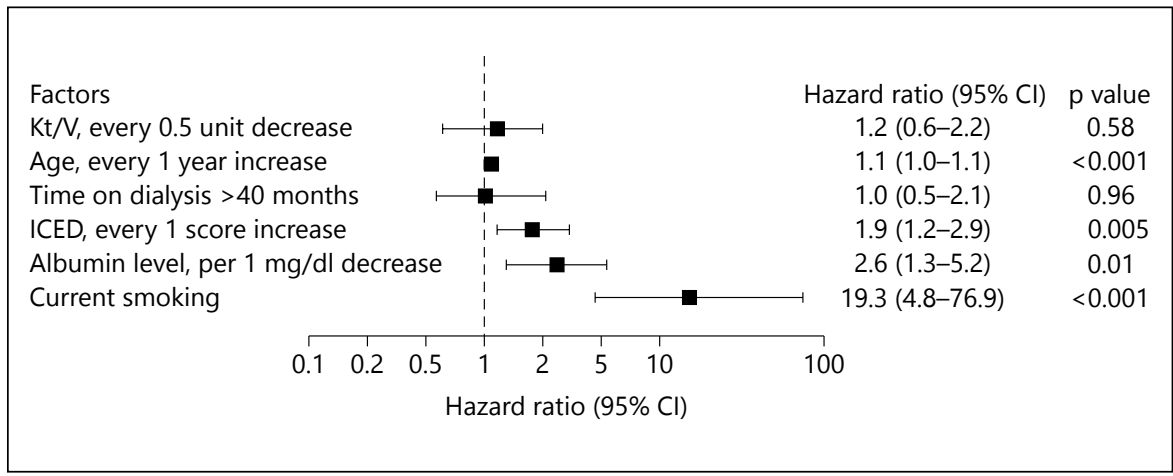

(95\% CI; 1.3 to $5.2, \mathrm{p}=0.01$ ), ICED, HR 1.9 for every 1 score increase ( $95 \% \mathrm{CI} ; 1.2$ to $2.9, \mathrm{p}=0.005)$, and age, HR 1.1 for every 1 year increase (95\% CI; 1.0 to $1.1, \mathrm{p}<0.001)$, figure 3. The model fitted well to the data (goodness of fit $\mathrm{p}=0.39$ ) and there was no departure from proportional hazard assumption $(\mathrm{p}=0.74)$.

\section{Discussion}

In this study, we demonstrated that the one-year morbidity and mortality rate of patients treated with twiceweekly HD and those of patients treated with thriceweekly HD are comparable. Among patients who were treated with twice-weekly HD, the level of spKt/V was not associated with one-year mortality. Four independent predictors of death were identified, including age, ICED, current smoking, and serum albumin level.

The NKF-DOQI HD Adequacy Work Group have recommended conventional thrice-weekly HD for all people who need dialysis therapy [1]. Twice-weekly HD is considered inadequate unless there is a reasonable amount of residual renal function. The recommended target $\mathrm{spKt} / \mathrm{V}$ is 1.2 and 2.0 (for patients with residual renal urea clearance of more than $2 \mathrm{ml} / \mathrm{min} / 1.73 \mathrm{~m}^{2}$ ) for thrice- and twice-weekly HD, respectively. The recommendation for twice-weekly HD was an extrapolation of urea kinetics in patients who were treated with thrice-weekly HD [1]. The extrapolation of effects of spKt/V on survival from studies among patients with thrice-weekly HD to patients with twice-weekly HD may not be rerated due to the differences in both salt accumulation and urea kinetics. Studies on the relationship between $\mathrm{spKt} / \mathrm{V}$ and survival in twice-weekly HD patients are limited. Recently, a 2-year cohort study from China demonstrated that the weekly spKt/V was a significant risk factor for death from a pooled analysis of patients dialyzed twice- or thrice- weekly HD [14]. Unfortunately, the study did not perform a subgroup analysis among patients who were on twice-weekly HD. In this study, we focused on patients with twice-weekly HD using a prospective design. Although the present study revealed a tendency for better survival among patients with higher $\mathrm{spKt} / \mathrm{V}$, we could not draw a firm conclusion about this because the difference in patient survival was not significant. No minimum cutoff point for $\mathrm{spKt} / \mathrm{V}$ was found in this study. The failure to demonstrate the effect of spKt/V on survival may be explained by the following reasons: first, spKt/V itself has little effect on short-term mortality, but may become more important in long-term dialysis; second, all patients might receive a far inadequate dosage of dialysis, no matter how 'high' or 'low' spKt/V was. The differences might be too small to show the effect of the twice-weekly HD dosage. The effect of spKt/V may be overshadowed by other risk factors, and a longer period of follow up may be needed to demonstrate this effect.

Our study has also demonstrated a comparable oneyear survival rate and hospitalization rate between patients with twice- and thrice-weekly HD. The finding was not unique. The survival rate for twice-weekly $\mathrm{HD}$ at one year in the present study was similar to that which has previously been reported from the study in China [14]. The study from China also demonstrated a comparable survival rate between patients treated with thrice-weekly and patients treated with twice-weekly dialysis, though the latter group were younger, had longer dialysis session time, higher spKt/V (per session) but shorter dialysis vintage [14]. A study from the USA also demonstrated a lower mortality risk for twice-weekly HD compared to those with thrice-weekly HD. However, the finding from the latter study probably resulted from a selection bias, because the patients with twice-weekly HD had greater residual renal function [6]. In our study, most of the patient's characteristics were comparable between the 
twice- and thrice-weekly HD groups, except for diabetes mellitus and duration of dialysis before recruitment. However, after adjustments for diabetes mellitus and duration of dialysis before recruitment, the survival rate for both groups were still comparable. As the majority of patients in twice-weekly HD group had been on dialysis for more than 1 year at the time of enrollment, one may speculate that the comparable survival rate between twiceand thrice-weekly HD might result from early death among patients with twice-weekly HD who did not take part in this study. This may have resulted in the selection of less severe cases for this cohort. However, our subgroup analysis demonstrated comparable one-year survival between the incident and prevalent groups among the twice-weekly HD patients making this unlikely. Therefore, our findings are less likely to result from a selection bias. It should be noted that the one-year survival rate in the present study, $94 \%$, was higher than what have been reported in few studies from the United States, ranging from 75.7 to $79.3 \%[15,16]$. The better survival rate would be explained by the relatively younger age, mean 57.8 years in this study, when compared to the United States mean age of 60.5 years [16]. The present study also excluded patients who died in the first 90 days. Another explanation was a lower severity of illness of the participants in this study. The proportion of ICED levels $0-1$, was $75 \%$ in this study, compared to that of the HEMO study, 35.1\% [9]. The better survival rate may also be explained by the racial difference, all Asian in this study, compare to the racial diversity of the US, $54 \%$ patients being white, $38.3 \%$ black, and $3.7 \%$ Asian $[16,17]$.

The morbidity determined by the probability of hospitalization in both groups was also comparable. The major causes of hospitalization were infections and cardiovascular disorders. The causes accounted for more than $50 \%$ of all hospitalizations. Our findings suggest that twice-weekly HD is not associated with a higher morbidity.

In this study, multiple potential risk factors of death were included in the analysis to identify predictors of death. The multivariable Cox regression model demonstrated that age, current smoking, serum albumin level, and ICED level were independent risk factors for death adjusted for time on HD and HD centers. Although age and ICED are significant, they are not correctable.

Hypoalbuminemia is a well-known risk factor in dialysis patients $[18,19]$. The association between low serum albumin and higher mortality may be explained by the concurrent inflammatory process [20] or by the consequences of poor nutritional status $[18,19,21]$. Smoking is considered a major risk factor for cardiovascular events in the general population. A prospective study has previously demonstrated an increased incidence of new-onset congestive heart failure, new-onset peripheral vascular diseases, and mortality among dialysis patients who smoke [22]. The increased risk of cardiovascular events may result from a higher level of fibrinogen and a higher systolic blood pressure in smokers than in non-smokers on dialysis [23]. A meta-analysis also showed a significantly higher risk of mortality in dialysis patients who smoke [24]. However, the higher mortality rate in the latter study is not associated with a higher prevalence of cardiovascular events [24]. Our study confirms that the patients who are current smokers are associated with increased mortality. The impact of smoking on mortality found in our study, $\mathrm{HR}=19.3$, is much higher than that reported previously. Our finding supports the K-DOQI guidelines advocating regular counseling and encouragement for ESRD patients to stop smoking [25]. Physicians should pay attention not only to spKt/V but also other modifiable factors associated with higher mortality in order to achieve a better patient outcome. Given that the negative impact of smoking on survival is so obvious within such a short follow up time, smoking cessation should be encouraged as soon as possible in all dialysis patients.

It should be pointed out that this study is associated with some limitations. First is the short follow-up period. An extrapolation of the result from this study to patients with longer dialysis duration may not be appropriated. Second, there was no determination of residual kidney function [RKF], which could have had an effect on patients' survival [26]. However, RKF is not routinely done in most dialysis centers. A prior study reported that the urine output based on self-report was reliable and was independently associated with lower all-cause mortality especially in participants who preserved urine output ( $>250 \mathrm{ml} /$ day) at one year of dialysis [27]. In this study, the self-report urine output was not significant and was removed from the model by the Cox regression analysis. Finally, as this study was not a randomized controlled trial, the findings should be considered suggestive rather than definitive. However, the study reflects real-world practice. Some potential confounders, such as prior duration of dialysis, dialysis centers, and the presence of diabetes, were adjusted for by using the multivariable Cox regression model.

The strength of the present study is its prospective design accounting for multiple known risk factors, including the index of coexistent disease. In addition, all relevant clinical events were conventionally censored using 
multivariable Cox regression analysis. Although there were 11 centers enrolled in this study, this was adjusted for in the final model.

\section{Conclusion}

Short-term survivals and hospitalizations for twiceand thrice-weekly dialysis were comparable. Among patients on twice-weekly HD, the effect of spKt/V on mortality was not obvious, whereas current smoking, hypoalbuminemia, old age, and high ICED were prominent prognostic factors for short-term mortality. Cessation of smoking and control of serum albumin level to within normal limits are crucial in clinical practice. Though conventional thrice-weekly HD is recommended based on international guideline, twice-weekly $\mathrm{HD}$ may be an acceptable alternative, short-term treatment scheme in resource-limited settings.

\section{Acknowledgments}

This study was supported by grants from the Royal College of Physician of Thailand, the Medical Association of Thailand, the Research and Training Center for Enhancing Quality of Life of
Working-Age People and the Kidney Foundation of Thailand. We gratefully thank the Northeastern Kidney Physician Network for both facilitating the research and for academic supports. We appreciate the Medical Research Foundation for allowing and supporting the use of the data management program, Online Medical Research Tool (OMERET). We sincerely thank Prof. Robert Mills and Gerald Kaye for their language revisions. We thank Saenhome W, RN, Piyasawatkul W, RN for study coordination of data acquisition and the study personnel of all sites for data gathering and outcomes record.

\section{Disclosure Statement}

None.

\section{Appendix}

Continuous variables that were categorized according to cutoff points that have been commonly used in previous studies included hemoglobin level $(<$ or $>10 \mathrm{gm} / \mathrm{dl})$, albumin level $(<$ or $>3.5 \mathrm{gm} / \mathrm{dl}$ ), potassium ( $>$ or $<5 \mathrm{mmol} / \mathrm{l}$ ), bicarbonate $(>$ or $<24$ $\mathrm{mmol} / \mathrm{l})$, calcium-phosphate index $(>$ or $<55)$, LDL cholesterol ( $>$ or $<100 \mathrm{mg} / \mathrm{dl}$ ), triglyceride ( $>$ or $<150 \mathrm{mg} / \mathrm{dl}$ ), ferritin level ( $>$ or $<500 \mathrm{ng} / \mathrm{ml}$ ), and nPCR $(<$ or $>1)$. The factors for which median values were used as cutoff points were epoietin dosage ( $>$ or $<8,000$ units per week), ICED score ( $>$ or $<1$ ), estimated GFR before $\mathrm{HD}\left(<\right.$ or $\left.>3.7 \mathrm{ml} / \mathrm{min} / 1.73 \mathrm{~m}^{2}\right)$, and serum creatinine $(<$ or $>11.7 \mathrm{mg} / \mathrm{dl})$.

\section{References}

1 Hemodialysis Adequacy 2006 Work Group: Clinical practice guidelines for hemodialysis adequacy, update 2006. Am J Kidney Dis 2006;48(suppl 1):S2-S90.

2 Port FK, Wolfe RA, Hulbert-Shearon TE, McCullough KP, Ashby VB, Held PJ: High dialysis dose is associated with lower mortality among women but not among men. Am J Kidney Dis 2004;43:1014-1023.

-3 Eknoyan G, Beck GJ, Cheung AK, Daugirdas JT, Greene T, Kusek JW, et al: Effect of dialysis dose and membrane flux in maintenance hemodialysis. N Engl J Med 2002;347:2010-2019.

-4 Termorshuizen F, Dekker FW, van Manen JG, Korevaar JC, Boeschoten EW, Krediet RT: Relative contribution of residual renal function and different measures of adequacy to survival in hemodialysis patients: an analysis of the Netherlands Cooperative Study on the Adequacy of Dialysis (NECOSAD)-2. J Am Soc Nephrol 2004;15:1061-1070.

5 Couchoud C, Kooman J, Finne P, Leivestad T, Stojceva-Taneva O, Ponikvar JB, et al: From registry data collection to international comparisons: examples of haemodialysis duration and frequency. Nephrol Dial Transplant 2009;24:217-224.
6 Hanson JA, Hulbert-Shearon TE, Ojo AO, Port FK, Wolfe RA, Agodoa LY, et al: Prescription of twice-weekly hemodialysis in the USA. Am J Nephrol 1999;19:625-633.

7 Athienites NV, Miskulin DC, Fernandez G, Bunnapradist S, Simon G, Landa M, et al: Comorbidity assessment in hemodialysis and peritoneal dialysis using the index of coexistent disease. Semin Dial 2000;13:320326.

-8 Miskulin DC, Martin AA, Brown R, Fink NE, Coresh J, Powe NR, et al: Predicting 1 year mortality in an outpatient haemodialysis population: a comparison of comorbidity instruments. Nephrol Dial Transplant 2004;19: 413-420.

-9 Miskulin DC, Athienites NV, Yan G, Martin AA, Ornt DB, Kusek JW, et al: Comorbidity assessment using the Index of Coexistent Diseases in a multicenter clinical trial. Kidney Int 2001;60:1498-1510.

10 Levey AS, Stevens LA, Schmid CH, Zhang YL, Castro AF 3rd, Feldman HI, et al: A new equation to estimate glomerular filtration rate. Ann Intern Med 2009;150:604-612.

11 Stevens LA, Schmid CH, Greene T, Zhang YL, Beck GJ, Froissart M, et al: Comparative per- formance of the CKD Epidemiology Collaboration (CKD-EPI) and the Modification of Diet in Renal Disease (MDRD) Study equations for estimating GFR levels above $60 \mathrm{~mL} /$ $\mathrm{min} / 1.73 \mathrm{~m} 2$. Am J Kidney Dis 2010;56:486495.

12 Schmoor C, Sauerbrei W, Schumacher M: Sample size considerations for the evaluation of prognostic factors in survival analysis. Stat Med 2000;19:441-452.

13 The Nephrology Society of Thailand: Thailand Renal Replacement Therapy Year 2008. Bangkok 2010.

14 Lin X, Yan Y, Ni Z, Gu L, Zhu M, Dai H, et al: Clinical outcome of twice-weekly hemodialysis patients in shanghai. Blood Purif 2012;33: 66-72.

15 USRDS 2012 Annual Report: National Institute of Diabetes and Digestive and Kidney Diseases. Bethesda, MD, 2012.

16 Goodkin DA, Bragg-Gresham JL, Koenig KG, Wolfe RA, Akiba T, Andreucci VE, et al: Association of comorbid conditions and mortality in hemodialysis patients in Europe, Japan, and the United States: the Dialysis Outcomes and Practice Patterns Study (DOPPS). J Am Soc Nephrol 2003;14:3270-3277. 
17 Cheng X, Nayyar S, Wang M, Li X, Sun Y, Huang W, et al: Mortality rates among prevalent hemodialysis patients in Beijing: a comparison with USRDS data. Nephrol Dial Transplant 2013;28:724-732.

18 Amaral S, Hwang W, Fivush B, Neu A, Frankenfield D, Furth S: Serum albumin level and risk for mortality and hospitalization in adolescents on hemodialysis. Clin J Am Soc Nephrol 2008;3:759-767.

19 Combe C, McCullough KP, Asano Y, Ginsberg N, Maroni BJ, Pifer TB: Kidney Disease Outcomes Quality Initiative (K/DOQI) and the Dialysis Outcomes and Practice Patterns Study (DOPPS): nutrition guidelines, indicators, and practices. Am J Kidney Dis 2004; 44(5 suppl 2):39-46.
20 de Mutsert R, Grootendorst DC, Indemans F, Boeschoten EW, Krediet RT, Dekker FW: Association between serum albumin and mortality in dialysis patients is partly explained by inflammation, and not by malnutrition. J Ren Nutr 2009;19:127-135.

21 Cooper BA, Penne EL, Bartlett LH, Pollock CA: Protein malnutrition and hypoalbuminemia as predictors of vascular events and mortality in ESRD. Am J Kidney Dis 2004;43:61-66.

22 Foley RN, Herzog CA, Collins AJ: Smoking and cardiovascular outcomes in dialysis patients: the United States Renal Data System Wave 2 study. Kidney Int 2003;63:1462-1467.

23 Biesenbach G, Zazgornik J: Influence of smoking on the survival rate of diabetic patients requiring hemodialysis. Diabetes Care 1996;19:625-628.

24 Liebman SE, Lamontagne SP, Huang LS, Messing S, Bushinsky DA: Smoking in dialysis patients: a systematic review and metaanalysis of mortality and cardiovascular morbidity. Am J Kidney Dis 2011;58:257-265.
25 K/DOQI Workgroup: K/DOQI clinical practice guidelines for cardiovascular disease in dialysis patients. Am J Kidney Dis 2005;45(4 suppl 3):S1-S153.

26 Vilar E, Wellsted D, Chandna SM, Greenwood RN, Farrington K: Residual renal function improves outcome in incremental haemodialysis despite reduced dialysis dose. Nephrol Dial Transplant 2009;24:25022510.

27 Shafi T, Jaar BG, Plantinga LC, Fink NE, Sadler JH, Parekh RS, et al: Association of residual urine output with mortality, quality of life, and inflammation in incident hemodialysis patients: the Choices for Healthy Outcomes in Caring for End-Stage Renal Disease (CHOICE) Study. Am J Kidney Dis 2010;56: 348-358. 